SPECIAL ISSUE

\title{
Telepsychiatry During the COVID Pandemic: Reflection from India
}

\author{
Harihara Suchandra', Dinakaran Damodaran', Barikar C. Malathesh', Lakshmi Nirisha \\ P.', Narayana Manjunatha', C Naveen Kumar', Gopi Gajera', Sujai Ramachandraiah', \\ Chethan Basavarajappa', Rajendra Gowda K.M. ${ }^{2}$ and Suresh Bada Math'* \\ 'Department of Psychiatry, NIMHANS, Bengaluru, Karnataka, India; '2Department of Child and Adolescent Psychiatry, \\ NIMHANS, Bengaluru, Karnataka, India
}

\section{Abstract}

Introduction: Recent advancements in information technology and access to smartphone have expanded the scope of healthcare delivery services across the globe. Telemedicine is making healthcare affordable and more accessible to the needy in situations like the present pandemic. Although telepsychiatry services were underutilised initially in India due to various barriers, its role in delivering healthcare services, has gained pace since the last few years. During the coronavirus disease (COVID-19) pandemic, India introduced telemedicine practice guidelines (March, 2020), and telepsychiatry operational guidelines (May, 2020), to remove barriers and promote equitable access. In the wake of COVID-19 pandemic various mental health institutes across India relied heavily upon telepsychiatry services to provide care. National Institute of Mental Health and Neuro Sciences, Bangalore being an Institute of National Importance has introduced different modules to provide clinical care to the mentally ill.

Objectives: In this article, the authors provide an experiential account of various clinical services provided by our institute through telepsychiatry means across India during the COVID-19 pandemic.

Conclusion: These clinical service modules have tremendous potential to increase the use of technology in providing quality care to the unreached population, bridging the treatment gap for psychiatric disorders globally and developing countries in particular.

Keywords: telepsychiatry; telemedicine practice guidelines; telepsychiatry operational guidelines; tele aftercare; telepsychiatry during pandemic

I nformation Technology has grown in leaps and bounds in the last two decades transforming human lives. Medical field is no exception to this revolution since it has seen significant innovations in research; however, there has been some resistance to applying newer information technology in clinical practice to provide services in the past. However, this pandemic, the subsequent lockdown and social distancing, has coerced the policymakers to innovate and devise various methods to reach out to a larger population (1). This technological upheaval is important to psychiatry as it can be a necessary vehicle to bridge the prevalent mental health treatment gap. In the ongoing coronavirus disease (COVID-19) pandemic, travel restrictions, inaccessibility to healthcare facilities and reduced in-person interactions has forced clinicians and service users to depend on telepsychiatry services heavily $(2,3)$.
India is the second most populated country in the world. India's population is equivalent to $17.7 \%(1.38$ billion) of the total world population. The Indian National Mental Health Survey of 2015-2016 has identified that the overall lifetime prevalence of mental disorder was $13.7 \%$ and cross-sectional prevalence was $10.6 \%$. The same study also highlighted that a treatment gap existed for mental disorders, ranging from 70 to $92 \%$ for different disorders (4). Also, World Health Organization (WHO) data shows that in India, there are only 0.29 psychiatrists per 1 lakh population, as opposed to 11.9 in high-income countries (5). With a majority of the psychiatrists in India being concentrated in urban centers, tertiary care hospitals and medical colleges in major cities, telepsychiatry will be an essential medium to reach the unreached $(6,7)$. 


\section{Telemedicine in India}

WHO defined telemedicine as the delivery of health-care services, where distance is a critical factor, by all healthcare professionals using information and communication technologies for the exchange of valid information for diagnosis, treatment and prevention of diseases, treating injuries, research and for the continuing education of health-care providers, all in the interests of advancing the health of individuals and their communities (8). Telepsychiatry can be considered as a sub-speciality under telemedicine, which can be used to provide specialised psychiatric services (9). Telepsychiatry can enrich access to mental health services, quality of care, community-based care and integration of psychiatric services into primary care while reducing the costs and logistic difficulties for service users $(3,10,11)$.

Indian Space Research Organization (ISRO) initiated a Telemedicine Pilot Project in the year 2001 which was implemented in several States across India with the objectives of bridging the health-care gap. However, due to inadequate human resources and technical support the intended objectives could not be met during that period (7), although the importance and benefits of telepsychiatry was understood across India. Recent literature highlighted that patient acceptance, clinical outcome, safety and satisfaction has been found to be similar to the conventional face-to-face consultation and care $(10,12)$. In the face of the ongoing COVID -19, the Board of Governors in supersession to the Medical Council of India, released 'Telemedicine Practice Guidelines-2020' on 25 March 2020 to enable telemedicine practice further and to provide an ethical and legal framework (13). Thus law, medicine and technology have finally caught up with the reality and the necessities of modern and challenging-pandemic times.

National Institute of Mental Health and Neuro Sciences (NIMHANS), Bengaluru being the centre for excellence in mental healthcare has identified the importance of telepsychiatry in the Indian set up and has designed various models to deliver clinical care. Following this, the Tele-psychiatry Operational Guidelines were framed by Indian Psychiatric Society, Telemedicine Society of India (TSI) and NIMHANS, Bengaluru empowering the psychiatrists to provide telepsychiatry services (9). In this article, the authors will be discussing about the various telemedicine services that are undertaken by the telepsychiatry center in NIMHANS.

\section{Tele-psychiatric aftercare (TAC) clinic: video based consultations}

Telemedicine Centre, NIMHANS, Bengaluru has been offering TAC services to those patients who have their treatment records at NIMHANS from 2016 (12). Patients are enrolled for this service from both outpatient and inpatient departments. Patients are selected by the treating team depending upon the convenience of the treating psychiatrist, following which the due procedure of obtaining the consent and fixing an appointment would be done. On the appointment day, staff from Telemedicine Centre will contact the patient and arrange for live video consultation with the treating psychiatrist. Following successful consultation, scanned copy of prescription will be sent through email or WhatsApp and the next appointment date is provided at the end of consultation. Telepsychiatry services have provided consultations successfully for the following number of patients: April 2018-March 2019: 288; April 2019-March 2020: 330; April 2020-Dec 2020: 933. There was a phenomenal increase in the number of telepsychiatry consultations provided during 2020, which can be directly attributed to lockdown restrictions. In addition, during the lockdown phase in India, our outpatient department services were closed for 2 months between March and May 2020. During this phase a total of 146 consultations were successfully provided, proving that this mode of service delivery was pandemic proof.

\section{Continuum of Care (COC): audio-based consultations}

The 'Continuum of Care' service was started between April and June 2020 during the COVID-19 pandemic, with the objective of extending healthcare and delivering medications at the patients' doorstep (for those who could not procure medicines). The Telemedicine Practice Guidelines of India recognises various modes (such as text, audio, video and combination) of communication that can be used by the doctors to provide healthcare services to the patients. Taking the advantage of this, a team of psychiatrists and psychiatry trainees reviewed files of pre-registered patients due for follow-up to collect their phone numbers, follow-up details and medication details. Telephonic calls were made to check the patient's status. For patients who were keeping well: e-prescriptions were sent to their mail address and WhatsApp; for those who were acutely ill, the NIMHANS team liaised with medical doctors and mental health professionals (MHPs) in Primary Health Centres (PHCs) and district hospitals for acute management. Around 15,000 patients were contacted telephonically and e-prescriptions/referral slips were sent. Among them our team identified around 1,600 patients who had difficulty in procuring medicines due to financial and conveyance difficulties, for whom free medicines were delivered at their doorsteps. Later in the feedback collected, most of the patients reported high satisfaction and acceptance for this patient-centered, clinician-led, telephonic follow-up. This model was adopted by many hospitals, medical colleges and other private health establishments to reach their patients during this pandemic. 
Samaashraya clinic: national helpline for psychosocial support and mental health services

The word 'Samaashraya' means 'seeking protection or common shelter'. This was an initiative from Ministry of Health and Family Welfare to address the pandemic related mental health concerns in India. This 24/7 helpline was started to combat the psychosocial crisis that people in the country were going through with the help of MHPs across the country. National Institute of Mental Health and Neuro Sciences was the first institute in India to start these services on 29th March 2020, which was later joined by other central (federal) government sponsored institutes. Gradually, these services included 661 MHPs from 20 states across India. A total of 333,854 calls were received and MHPs provided services to 52,352 callers. Services were provided in 13 different vernacular Indian languages through dedicated channels for Child, Adult, Elderly, Women and Healthcare worker. A preliminary feedback from the callers, done after 2 weeks of the service initiation revealed that over $90 \%$ callers were satisfied with the provision of the service (14).

\section{Interactive voice response system (IVRS)}

As the Government began to relax the lockdown measures hospitals, medical colleges, mental hospitals, private health establishments, out-patient clinics and NIMHANS resumed their OPD services. With the objective of reducing overcrowding and maintaining social distance norms many health establishments initiated IVRS services. Patients can approach the online helpline to book OPD appointments and follow-up consultations. Within a month at NIMHANS, 2,673 patients had utilised the IVRS services.

\section{Mental health services for quarantined air travellers}

Government of India made it mandatory for all international air travellers to be in 14 days of quarantine following their international travel. It was noted that there was lot of anxiety, stigma and fear of getting infected among them. Upon the advice of Government of Karnataka, NIMHANS formed a team of Psychiatrists to contact the quarantined international air travellers and resolve mental health related issues telephonically. A total of 1,600 people in the quarantine homes were contacted. Major issues noted among them were anxiety and adjustment disorders. Psychological first aid was done as per disaster protocol management provided by WHO (15).

\section{Policymaking}

India's digital health policy advocates the use of digital tools for improving the efficiency and outcomes of the healthcare systems and lays significant focus on the use of tele-services. During the COVID-19 pandemic, NIMHANS was extensively involved in the policymaking for tele services in collaboration with Government of India.
On par with western countries like the USA and the UK, India also developed various tele related guidelines for psychiatry, psychotherapy, psychiatric social work (PSW) and nursing.

\section{Tele-medicine practice guidelines}

The Indian Government has published Telemedicine Practice Guidelines on March 25, 2020. These guidelines clarify India's position on tele-consultation and is legal to provide tele-services by registered medical practitioners in line with the requirements of the Telemedicine Guidelines (13). The notification of the Telemedicine Practice Guidelines marks the dawn of a new era in the practice of medicine. This also triggered the complementary alternative systems, releasing their Telemedicine Practice Guidelines for AYUSH (Ayurveda, Unani and Siddha) practitioners on 7 April 2020 by the Central Council of Indian Medicine. On similar lines, a telemedicine practice guideline was released on 10 April 2020 by the Central Council of Homeopathy, enabling registered homoeopathic practitioners to continue with their practice. In this regard, it is encouraging that the Government of India has been very progressive in introducing information technology not only for modern medicine, but also for the alternative systems of medicine so that the patient has various options to choose from for their health needs, and a comprehensive effort is made to reach the unreached.

\section{Telepsychiatry Operational Guidelines ( I 6)}

The Telemedicine Society of India \& Indian Psychiatric Society in association with NIMHANS, Bengaluru (an Institution of National Importance), undertook this effort to bring in the Telepsychiatry Operational Guidelines (16). This guideline was discussed and widely accepted, although it had its critics who voiced their reservations regarding the central government prohibiting the use of telemedicine to prescribe habit-forming drugs. However, these guidelines were later amended by the Government of India allowing practitioners to prescribe Clonazepam, Clobazam and Phenobarbitone. The purpose of the telepsychiatry operational guideline is to give practical advice to psychiatrists so that telepsychiatry services become a part of normal day-to-day clinical practice. The main aim of this guide is to assist, educate and provide guidance to psychiatrists in setting up, implementation, administration and provision of telepsychiatry service. This is not a standalone document and should be used in conjunction with other national clinical standards, protocols, policies, procedures, laws, ethical regulations and the Telemedicine Practice Guidelines 2020, notified by the Ministry of Health and Family Welfare, New Delhi. The psychiatrists were advised to read the guidelines in conjunction 
with other laws such as the Mental Healthcare Act 2017, The Rights of Persons with Disability Act 2016, Narcotic Drugs and Psychotropic Substances Act 1985, Drugs and Cosmetics Act, 1940 and Information and Technology Act 2000.

\section{NIMHANS - tele-psychotherapy practice guidelines}

The COVID-19 pandemic has resulted in the disruption of continued psychological services to our clients and has come as a barrier for persons seeking to engage with professional psychotherapy services. In this context, the Department of Clinical Psychology at NIMHANS, has taken the initiative to formulate Guidelines for Tele-psychotherapy Services. These guidelines aim to standardise competent and ethical tele-psychotherapy services in our country to benefit clients in need, while safeguarding their interests. These guidelines have been developed in response to the emergent needs of access and continuity of psychotherapy services during the pandemic. This guideline was formulated after referring to a range of national and international practice guidelines for telepsychology and telepsychiatry. The contextual realities of the practice of psychotherapy in India have been considered in the preparation of these guidelines (17). This guideline gave ethical and legal framework to provide counselling services across the country.

\section{Telepsychiatric social work practice guidelines}

The department of Psychiatric Social Work (PSW) is an integral part of the multidisciplinary team at NIMHANS and has been instrumental in providing PSW services. The use of technological tools in PSW practice may be offered as a remote service, or in conjunction with in-person PSW services. These guidelines are developed in response to the emergent needs of access and continuity of PSW services during the COVID-19 pandemic. However, the guidelines are not restricted to any particular time period and aims to assist PSWs, who are working in mental health settings. Contemporary PSWs can provide services to individual clients, groups and communities by using online/telephonic/video/self-guided web-based services. Psychiatric social work practice using telecommunication technology includes the use of email, text messaging, video conferencing, online chat, or internet phone calls. The following standards are provided for five main PSW services, which are: (1) working with individuals, (2) working with families, (3) working with groups, (4) crisis management, and (5) working with communities. Each practice standard provides PSWs with a general guidance on how to use technological tools ethically and maintain the professional boundary. The standards and their interpretations are intended to set a minimum score of excellence for professional practice when PSWs use technology, and to provide a framework to address possible benefits, challenges and risks that arise when using these tools in the practice of tele-PSW services across the country (18).

\section{NIMHANS - telenursing practice guidelines}

In line with the above, NIMHANS nursing professionals in association with TSI and Trained Nurses Association of India (TNI) drafted NIMHANS Telenursing Practice Guidelines for providing nursing services using technology to reach the unreached (19). The purpose of this tele-nursing document is to provide general guidelines to registered nurses about the use of telehealth technology as a part of day-to-day nursing practice. These guidelines help registered nurses in identifying their roles and responsibilities to provide safe, compassionate, competent and ethical care. These telenursing guidelines were developed based on the code of ethics and professional conduct for nurses in India. Therefore, it provides a clear direction for registered nurses to practice telenursing within their professional boundaries. These guidelines also help the nurses on how to collaborate with specialist doctors, professional colleagues and other healthcare team members in offering the best possible care in a timely manner to patients. However, these guidelines should be used in conjunction with other national clinical standards, protocols, policies, ethics, laws and procedures.

\section{Advisory on tele-yoga services (20)}

Yoga is successfully applied in various psychiatric disorders and also in maintaining positive mental health/ well-being over the globe with encouraging evidence coming from scientific publications in reputed journals, especially in the last three decades. These reports suggest a need to integrate yoga in mental health services (21). Department of Integrative Medicine, National Institute of Mental Health and Neurosciences (NIMHANS), Bengaluru was providing tele-yoga services during this pandemic to persons with mental illness, caregivers and for healthcare workers to reduce stress and inculcate positive well-being. This is the first, one-of-its-kind department in an Institute of National Importance in India, which integrates traditional Indian knowledge from Ayurveda and Yoga, with modern medical science in providing clinical services, academic training and research activities with special focus on mental health and neurosciences. This department provided tele-yoga services on a daily basis during this pandemic. Based on their vast experience, the Advisory on Tele-Yoga Services (20) was released on 21 July 2020. The Central Council for Research in Yoga and Naturopathy (CCRYN), Ministry of AYUSH, Government of India, New Delhi in collaboration with Department of Integrative Medicine, 
National Institute of Mental Health and Neurosciences (NIMHANS), and Swami Vivekananda Yoga Anusandhana Samsthana (S-VYASA), Bengaluru conceptualised, drafted and released this Tele-Yoga Advisory Guidelines.

\section{Future considerations}

For telehealth to be effective during the current COVID19 pandemic and also to become part of the day-to-day practice in future, it needs to be appropriately integrated into health services. To make telehealth a mainstream component of the health system, the authors propose the following measures:

- To adopt, adapt and amalgamate the tele-mental health services in the national mental health programme

- Adequate education and training of all health professionals in telemedicine

- Adequate funding to cover the cost of delivering telepsychiatry, telenursing and tele-mental health services

- Restructure clinical models of patient care

- To integrate psychiatry with community care through telepsychiatry services

- To incentivise the tele-mental health services

- To make technological advances and provide access to modern information technology in rural area

- To adopt technology as the main tool to provide healthcare at the doorsteps through planned 'Health \& Wellness Centres' across the country

- To remove all bottle necks in implementing tele-mental health services across the nation

- To encourage and invest research in the area of artificial intelligence and machine learning in the public mental health

\section{Conclusion}

The COVID-19 crisis and global pandemic may be the defining moment for the digital mental health. One of the definite solutions to continue delivering mental health support within this pandemic from a safe distance is telepsychiatry. According to our experience, telepsychiatry services are realistically possible and apt to help patients and caregivers not just during the pandemic, but also in the future. This would help in decreasing the inflow of regular OPD consultations and minimising the chances of exposure to novel coronavirus. It would also save transport and other logistical costs to the patients and their caregivers in the future. Telepsychiatry is also a useful tool for integrating psychiatry in primary healthcare. Mental illnesses are going to be a leading public health challenge during and after the pandemic. There exist colossal diagnostic and treatment gaps in psychiatry that in themselves become barriers for access to treatment. These challenges can be addressed to some extent by comprehensively adopting telepsychiatry.

\section{References}

1. Manjunatha N, Kumar CN, Math SB. Coronavirus disease 2019 pandemic: time to optimize the potential of telepsychiatric aftercare clinic to ensure the continuity of care. Indian J Psychiatry 2020; 62(3): 320-1. doi: 10.4103/psychiatry.IndianJPsychiatry_236_20

2. Naik SS, Manjunatha N, Kumar CN, Math SB, Moirangthem S. Patient's perspectives of telepsychiatry: the past, present and future. Indian J Psychol Med 2020 Oct 1; 42(5_suppl): 102S-7S. doi: 10.1177/0253717620963341

3. Ibrahim FA, Pahuja E, Dinakaran D, Manjunatha N, Kumar $\mathrm{CN}$, Math SB. The future of telepsychiatry in India. Indian J Psychol Med 2020 Oct; 42(5_suppl): 112S-17S. doi: $10.1177 / 0253717620959255$

4. Gautham MS, Gururaj G, Varghese M, Benegal V, Rao GN, Kokane A, et al. The National Mental Health Survey of India (2016): prevalence, socio-demographic correlates and treatment gap of mental morbidity. Int J Soc Psychiatry 2020 Jun; 66(4): $361-72$.

5. World Health Organization, editor. Mental health atlas 2017. Geneva, Switzerland: World Health Organization; 2018, 62 p.

6. Gowda GS, Noorthoorn EO, Lepping P, Kumar CN, Nanjegowda RB, Math SB. Factors influencing advance directives among psychiatric inpatients in India. Int J Law Psychiatry 2018 Feb; 56: 17-26. doi: 10.1016/j.ijlp.2017.10.002

7. Math SB, Moirangthem S, Kumar NC. Tele-psychiatry: after mars, can we reach the unreached? Indian J Psychol Med 2015 Apr; 37(2): 120-1. doi: 10.4103/0253-7176.155606

8. Telemedicine Facility. Available from: https://www.aiims.edu/aiims/ telemedicine/telepage_ch_23_10_17.htm [cited 15 December 2020].

9. Dinakaran D, Basavarajappa C, Manjunatha N, Kumar $\mathrm{CN}$, Math SB. Telemedicine practice guidelines and telepsychiatry operational guidelines, India - a commentary. Indian J Psychol Med 2020 Sep 25; 42(5_suppl): 1S-3S. doi: $10.1177 / 0253717620958382$

10. Warmerdam L, Smit F, van Straten A, Riper H, Cuijpers P. Cost-utility and cost-effectiveness of internet-based treatment for adults with depressive symptoms: randomized trial. J Med Internet Res 2010 Dec 19; 12(5): e53. doi: 10.2196/jmir.1436

11. Kumar MS, Krishnamurthy S, Dhruve N, Somashekar B, Gowda MR. Telepsychiatry netiquette: connect, communicate and consult. Indian J Psychol Med 2020 Oct 1; 42(5_suppl): 22S-6S. doi: 10.1177/0253717620958170

12. Das S, Manjunatha N, Kumar CN, Math SB, Thirthalli J. Tele-psychiatric after care clinic for the continuity of care: a pilot study from an academic hospital. Asian J Psychiatry 2020 Feb; 48: 101886. doi: 10.1016/j.ajp.2019.101886

13. Telemedicine.pdf. Available from: https://www.mohfw.gov.in/ pdf/Telemedicine.pdf [cited 15 December 2020].

14. Ravindran S, P Nirisha L, Channaveerachari NK, Seshadri SP, Kasi S, Manikappa SK, et al. Crossing barriers: role of a tele-outreach program addressing psychosocial needs in the midst of COVID-19 pandemic. Asian J Psychiatry 2020 Oct; 53: 102351. doi: 10.1016/j.ajp.2020.102351

15. Ritish D, Dinakaran D, Chander R, Murugesan M, Ibrahim FA, Parthasarathy R, et al. Mental health concerns in quarantined international air passengers during COVID-19 pandemic - an experiential account. Asian J Psychiatry 2020 Oct; 53: 102364. doi: 10.1016/j.ajp.2020.102364 
16. Math SB, Manjunatha N, Kumar CN, Chethan B, Gangadhar BN. Telepsychiatry operational guidelines-2020. Bengaluru: NIMHANS; 2020. Telepsychiatry-OperationalGuidelines-2020-Inaug.pdf. Available from: http:// nimhans.co.in/wp-content/uploads/2020/06/TelepsychiatryOperational-Guidelines-2020-Inaug.pdf [cited 22 December 2020].

17. Guidelines-for-Telepsychotherapy-Services-17.4.2020.pdf. Available from: https://nimhans.ac.in/wp-content/uploads/2020/04/ Guidelines-for-Telepsychotherapy-Services-17.4.2020.pdf [cited 18 December 2020].

18. Guidelines for tele - psychiatric social work practice. Available from: https://nimhans.ac.in/wp-content/uploads/2020/
04/Guidelines-for-Telepsychiatry-social-work-Inaug.pdf [cited 18 December 2020].

19. Poreddi V, Kathyayani B, Hatti NM, Konduru R, Manjunatha N, Kumar N, et al. NIMHANS telenursing practice guidelines 2020. 2020. Available from: https://nimhans.ac.in/wp-content/ uploads/2020/12/TeleNursing-Practice-Guideline-2020.pdf [cited 20 December 2020].

20. Advisory on Tele-Yoga Services. Available from: https://main. ayush.gov.in/sites/default/files/advisory $\% 20$ on $\% 20$ tele $\% 20$ yoga\%20services\%202020_0.pdf [cited 24 December 2020].

21. Gangadhar BN, Varambally S. Yoga as therapy in psychiatric disorders: past, present, and future. Biofeedback 2011; 39: 60-3. doi: 10.5298/1081-5937-39.2.03 